\title{
A Three Degree-of-Freedom Weakly Coupled Resonator Sensor With Enhanced Stiffness Sensitivity
}

\author{
Chun Zhao, Student Member, IEEE, Graham S. Wood, Member, IEEE, Jianbing Xie, Member, IEEE, \\ Honglong Chang, Member, IEEE, Suan Hui Pu, Member, IEEE, and Michael Kraft
}

\begin{abstract}
This paper reports a three degree-offreedom (3DoF) microelectromechanical systems (MEMS) resonant sensing device consisting of three weakly coupled resonators with enhanced sensitivity to stiffness change. If one resonator of the system is perturbed by an external stimulus, mode localization occurs, which can be detected by a change of modal amplitude ratio. The perturbation can be, for example, a change in stiffness of one resonator. A detailed theoretical investigation revealed that a mode aliasing effect, along with the thermal noise floor of the sensor and the associated electrical system ultimately limit the dynamic range of the sensor. The nonlinearity of the 3DoF sensor was also analyzed theoretically. The $3 D$ DoF resonator device was fabricated using a silicon on insulator process. Measurement results from a prototype device agreed well with the predictions of the analytical model. A significant, namely 49 times, improvement in sensitivity to stiffness change was evident from the fabricated 3DoF resonator sensor compared with the existing state-of-the-art 2DoF resonator sensors, while the typical nonlinearity was smaller than $\pm 2 \%$ for a wide span of stiffness change. In addition, measurements indicate that a dynamic range of at least $39.1 \mathrm{~dB}$ is achievable, which could be further extended by decreasing the noise of the device and the interface electronics.

[2015-0020]
\end{abstract}

Index Terms-Microelectromechanical systems (MEMS) resonator, stiffness change sensor, dynamic range, nonlinearity, three degree-of-freedom.

\section{INTRODUCTION}

$\mathbf{O}$ VER THE last couple of decades, micro- and nanofabricated resonant devices have been widely used to sense small changes in the properties of the resonator [1], namely the stiffness [2] and mass [3] of the resonator. Among these, sensing devices that detect stiffness change have been

Manuscript received January 19, 2015; revised September 3, 2015; accepted October 11, 2015. Subject Editor G. Piazza.

C. Zhao and G. S. Wood are with the Nano Research Group, School of Electronics and Computer Science, University of Southampton, Southampton SO17 1BJ, U.K. (e-mail: cz1y10@ecs.soton.ac.uk; gsw1g11@ecs.soton.ac.uk).

J. Xie and H. Chang are with the Key Laboratory of Micro and Nano Systems for Aerospace, Ministry of Education, Northwestern Polytechnical University, Xi'an 710072, China (e-mail: xiejb@nwpu.edu.cn; changhl@nwpu.edu.cn).

S. H. Pu is with Nano Research Group, School of Electronics and Computer Science, University of Southampton, Southampton SO17 1BJ, U.K., and also with the University of Southampton Malaysia Campus, Nusajaya 79200, Malaysia (e-mail: suanhui.pu@southampton.ac.uk).

M. Kraft is with the Montefiore Institute, University of Liège, Liège 4000, Belgium (e-mail: mk1@ecs.soton.ac.uk).

Color versions of one or more of the figures in this paper are available online at http://ieeexplore.ieee.org.

Digital Object Identifier 10.1109/JMEMS.2015.2490204 employed for many applications, including accelerometers [4], strain sensors [5], pressure sensors [6] and force gradient sensors for imaging microscopy [7], [8]. So far, practically all of these resonant sensors utilize a frequency shift, caused by stiffness change due to the quantity to be measured, as the output signal, which has the advantage that it is a quasi-digital signal [4].

Recently, an alternative sensing approach has been proposed [9]-[11]. By adding an identical resonator and a weak coupling element between to form a 2 degree-offreedom (DoF) resonator system, it has been shown that the sensitivity can be improved by orders of magnitude compared to the conventional frequency modulation approach in a device of similar size and fundamental resonant frequency [9], [10]. The principle of operation of these devices is based on a well-known phenomenon called mode localization, which was first described in solid-state physics by Anderson [12]. When a structural perturbation (e.g. mass or stiffness change) is introduced into a coupled resonator system, the propagation of the vibration is disrupted, leading to an alteration in the linear mode shapes of the system.

Due to the enhanced sensitivity, 2DoF devices have been used in various sensing applications such as mass sensing [9] and stiffness change sensing, including but not limited to an electrometer [13] and a displacement sensor [14]. Similar concept has also been used for a novel capacitive readout [15]. The coupling element between the resonators can be divided into two categories, mechanical coupling [9] and electrostatic coupling [16]. The electrostatic coupling has the advantage of being controllable by tuning the electrical potential difference across the coupling element [16].

Attempts have also been made to extend the number of identical coupled resonators even further, to a number of 15 [17]. The intended application of the device is analyte mass sensing and to identify on which resonator the analyte has docked. In terms of sensitivity, it demonstrated an order of magnitude improvement in mass sensitivity comparing to the $2 \mathrm{DoF}$ resonator system.

In the work presented in the following, we use a different approach based on three weakly coupled resonators (i.e. a $3 \mathrm{DoF}$ system), in which the stiffness of the resonator in the middle is at least twice the value compared to the other two identical resonators. A schematic drawing of the prototype device to be investigated in this work is shown in Fig. 1. Each resonator consists of four suspension beams and a proof 


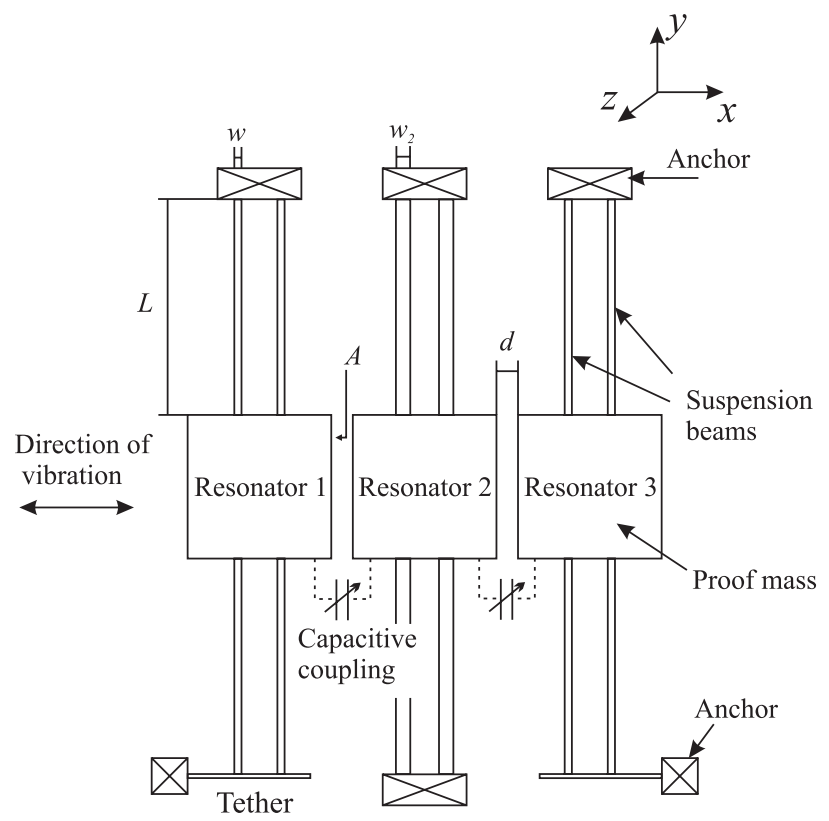

Fig. 1. Schematic drawing of the prototype $3 \mathrm{DoF}$ resonator sensing device used in this work.

mass, while the outer resonators (i.e. resonators 1 and 3) have tethers attached to facilitate axial force sensing [18], [19]. However, the tethers were designed to be stiff in the $x$-axis. Because of this, in conjunction with the high stiffnesses of the suspension beams in the $y$-axis, it can be assumed that the suspension beams are anchored at the tethers when the vibration displacement of the proof masses are small (less than $1 \mu \mathrm{m}$ in our study). The device is intended for sensing a change in stiffness in this work. With the $3 \mathrm{DoF}$ resonator sensing device, an order of magnitude improvement in the stiffness sensitivity could be demonstrated.

Furthermore, previous studies concentrated on the sensitivity for a change in mass or stiffness only within a narrow range; therefore other characteristics of coupled resonator sensors such as linearity and dynamic range have not yet been investigated. In the work presented in the following, by adopting an alternative analytical approach using transfer function models [19], we analyze the nonlinearity and dynamic range of a sensing device consisting of three coupled resonators.

The paper begins with a theoretical analysis of the $3 \mathrm{DoF}$ resonator sensor based on the transfer function model in section II. The theoretical sensitivity and dynamic range of the sensor are also analyzed in this section. Then, a description of the device, including fabrication process and device design, is given in section III, followed by a description of the experimental set up and methodology in section IV. The measurement results and their discussion are presented in section $\mathrm{V}$ and finally the paper ends with conclusions in section VI.

\section{THEORY}

\section{A. 3DoF Resonator System Model}

In order to understand the physics of the $3 \mathrm{DoF}$ resonator system shown in Fig. 1, consider the lumped parameter block diagram of a three coupled resonator system

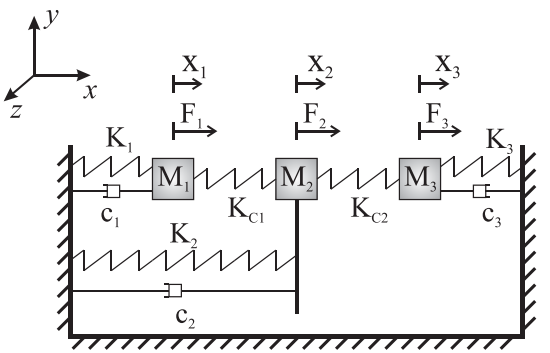

Fig. 2. Mass-damper-spring lumped parameter model of a 3DoF resonator sensing device.

as shown in Fig. 2. Each resonator consists of a mass, spring and damper, and is coupled to its neighboring resonator through springs $\left(K_{c 1}\right.$ and $\left.K_{c 2}\right)$. Since the device operated in vacuum in our study, damping of each individual resonator is dominated by energy loss mechanisms such as anchor loss over gas damping [20]; whereas damping between the masses is dominated by the gas damping between the parallel plates, which becomes negligible under vacuum ambient pressure compared to that of each individual resonator, and thus is not included.

Suppose the masses of all resonators and their corresponding coupling spring stiffnesses are identical, i.e., $M_{1}=M_{2}=$ $M_{3}=M$ and $K_{c 1}=K_{c 2}=K_{c}$, while the spring stiffnesses of the outer resonators are asymmetrical with $K_{1}=K$, $K_{3}=K+\Delta K(\Delta K$ is the stiffness perturbation due to a physical quantity to be measured), and the stiffness of the resonator in the middle is different from the other two resonators. The damping coefficients are neglected for much of the analysis that follows, but are included in Fig. 2 for reference in later sections. Further, assuming linear springs, no movement in the $y$ and $z$-axis, and the system is driven from only one side, namely with $F_{1}(s)=F(s)$ and $F_{2}(s)=F_{3}(s)=0$, the displacement responses $X_{1}(s), X_{3}(s)$ can be derived with respect to $F(s)$, from the equations of motion given by [19]:

$$
\begin{aligned}
\frac{X_{1}(s)}{F(s)} & =\frac{H_{2}(s) H_{3}(s)-K_{c}^{2}}{H_{1}(s) H_{2}(s) H_{3}(s)-\left[H_{1}(s)+H_{3}(s)\right] K_{c}^{2}} \\
\frac{X_{3}(s)}{F(s)} & =\frac{K_{c}^{2}}{H_{1}(s) H_{2}(s) H_{3}(s)-\left[H_{1}(s)+H_{3}(s)\right] K_{c}^{2}}
\end{aligned}
$$

where the particular transfer functions, $H_{i}(s), i=1,2$ and 3 , are defined as [19]:

$$
\begin{aligned}
& H_{1}(s) \equiv M s^{2}+c_{1} s+\left(K+K_{c}\right) \\
& H_{2}(s) \equiv M s^{2}+c_{2} s+\left(K_{2}+2 K_{c}\right) \\
& H_{3}(s) \equiv M s^{2}+c_{3} s+\left(K+K_{c}+\Delta K\right)
\end{aligned}
$$

Ideally, with negligible damping and $\Delta K=0$, there are three distinctive modes of the system: in the first mode with the lowest frequency, the three resonators vibrate in-phase; in the second mode, the resonator in the middle remains stationary while the resonators on either sides vibrate out-of-phase, with a phase difference of $180^{\circ}$; in the third mode having the highest resonant frequency, each resonator is out-of-phase with its neighboring resonator, so the left and right resonators are in phase, but out-of-phase with the resonator in the center [21]. When a perturbation occurs 
in stiffness with $\Delta K \neq 0$, all three modes are disturbed; i.e. both the mode shapes and frequencies change (the order of the modes in the frequency domain remains unchanged due to frequency veering [22]). Due to the stiffness perturbation, the vibrational energy within the system becomes locally confined; hence the mode amplitude pattern of the system changes, and mode localization occurs [22]. The modes of interest for the purpose of this work are the first two modes, which we will refer to as the in-phase and out-of-phase modes, respectively.

\section{B. Analysis Without Damping}

1) Amplitude Ratio Without Damping: A recent comparative study [23] showed that amplitude ratio can be the optimum way of measuring the effect of mode localization in terms of linear sensitivity and linearity, compared to eigenstates shift [9], [10]. Hence we shall use amplitude ratio as the output quantity, given by:

$$
\text { Output }=\left|\frac{X_{1}(s)}{X_{3}(s)}\right|
$$

Assuming a weak coupling stiffness, $K_{c}<K / 10$ and the stiffness of the resonator in the middle being more than twice that of resonators 1 and $3, K_{2}>2 K$, the following condition is satisfied:

$$
K_{c}<\frac{K}{10}<\frac{K_{2}-K}{10}
$$

Furthermore, assuming no damping is present in the system, so the quality factor $Q \rightarrow \infty$, and $\Delta K \ll K$, let $s=j \omega$, the frequencies of the in-phase and out-of-phase modes can be derived as [24]:

$$
\omega_{\text {ip }} \approx \sqrt{\frac{1}{M}\left[K+K_{c}+\frac{1}{2}\left(\Delta K-\frac{2 K}{\gamma}-\sqrt{\left.\Delta K^{2}+\left(\frac{2 K}{\gamma}\right)^{2}\right)}\right]\right.}
$$

$$
\omega_{\mathrm{op}} \approx \sqrt{\frac{1}{M}\left[K+K_{c}+\frac{1}{2}\left(\Delta K-\frac{2 K}{\gamma}+\sqrt{\left.\Delta K^{2}+\left(\frac{2 K}{\gamma}\right)^{2}\right)}\right]\right.}
$$

where $\omega_{\mathrm{ip}}$ and $\omega_{\mathrm{op}}$ denote the frequencies of the in-phase and out-of-phase modes, respectively, and

$$
\gamma=\frac{K\left(K_{2}-K+K_{c}\right)}{K_{c}^{2}}
$$

Substituting (8) and (9) into (1) and (2), the amplitude ratios for the in-phase and out-of-phase modes, $\left|X_{1}\left(j \omega_{\text {ip }}\right) / X_{3}\left(j \omega_{\text {ip }}\right)\right|$ and $\left|X_{1}\left(j \omega_{\mathrm{op}}\right) / X_{3}\left(j \omega_{\mathrm{op}}\right)\right|$, respectively, can be estimated as [24]:

$$
\begin{aligned}
& \left|\frac{X_{1}\left(j \omega_{\mathrm{ip}}\right)}{X_{3}\left(j \omega_{\mathrm{ip}}\right)}\right|_{Q \rightarrow \infty} \approx\left|\frac{\sqrt{\gamma^{2}(\Delta K / K)^{2}+4}+\gamma(\Delta K / K)}{2}\right| \\
& \left|\frac{X_{1}\left(j \omega_{\mathrm{op}}\right)}{X_{3}\left(j \omega_{\mathrm{op}}\right)}\right|_{Q \rightarrow \infty} \approx\left|-\frac{\sqrt{\gamma^{2}(\Delta K / K)^{2}+4}-\gamma(\Delta K / K)}{2}\right|
\end{aligned}
$$

TABLE I

Values Used in the Simulation (Without Damping)

\begin{tabular}{l|l|l}
\hline Component & Value & Remarks \\
\hline$M$ & $6.94 \times 10^{-9} \mathrm{~kg}$ & Masses of the three resonators \\
\hline$K$ & $55.73 \mathrm{~N} / \mathrm{m}$ & Stiffnesses of the outer resonators \\
\hline$K_{2}$ & $167.20 \mathrm{~N} / \mathrm{m}$ & $\begin{array}{l}\text { Stiffness of the middle resonator, } \\
K_{2} / K=3\end{array}$ \\
\hline \multirow{2}{*}{$K_{c}$} & $1.11 \mathrm{~N} / \mathrm{m}$ & $\begin{array}{l}\text { Coupling stiffness, } K / K_{c}=50, \\
\gamma=5050\end{array}$ \\
\cline { 2 - 3 } & $0.74 \mathrm{~N} / \mathrm{m}$ & $\begin{array}{l}\text { Coupling stiffness, } K / K_{c}=75, \\
\gamma=11325\end{array}$ \\
\hline \multirow{2}{*}{$\omega_{0}$} & $14.27 \mathrm{kHz}$ & $\begin{array}{l}\text { Resonant frequency } \\
\text { of single resonator (outer) }\end{array}$ \\
\hline
\end{tabular}

To verify the results, since damping is neglected, the mode frequencies and amplitude ratios can be obtained using Matlab by solving the eigenvalue problem [25] of the 3DoF system, which can be expressed in the form of (13). The mode frequencies are the square roots of the eigenvalues of the mass normalized stiffness matrix (i.e. $\omega=\sqrt{\lambda}$ ), while the amplitude ratios can be obtained from the eigenstates $\left(\left[\begin{array}{lll}x_{1} & x_{2} & x_{3}\end{array}\right]^{\mathrm{T}}\right)$. The $\gamma$ value was 11325 for the frequency simulation. The values of other stiffnesses and the mass chosen for the simulation are listed in Tab. I. All values have been chosen to be close to the actual fabricated device.

$$
\lambda\left[\begin{array}{l}
x_{1} \\
x_{2} \\
x_{3}
\end{array}\right]=\left[\begin{array}{ccc}
\frac{K+K_{c}}{M} & -\frac{K_{c}}{M} & 0 \\
-\frac{K_{c}}{M} & \frac{K_{2}+2 K_{c}}{M} & -\frac{K_{c}}{M} \\
0 & -\frac{K_{c}}{M} & \frac{K+\Delta K+K_{c}}{M}
\end{array}\right]\left[\begin{array}{l}
x_{1} \\
x_{2} \\
x_{3}
\end{array}\right]
$$

Fig. 3a verifies the expression of frequencies of each mode, i.e. (8) and (9), as the simulated mode frequencies match very well with theoretical calculations, with error smaller than 10ppm, thus justifying the simplifying assumptions. Fig. $3 \mathrm{~b}$ shows the simulated and theoretically calculated values of the mode frequency difference as a function of normalized stiffness perturbation. It can be seen from Fig. $3 b$ that the frequency difference first decreases with increasing (negative) stiffness perturbation, then increases symmetrically with increasing (positive) stiffness perturbation. As expected, the frequency difference is always positive, indicating that the out-of-phase mode always has a higher frequency than the in-phase mode regardless of the stiffness perturbation.

Fig. 4 shows the amplitude ratios as a function of normalized stiffness perturbation. It is found from Fig. 4 that the theoretical estimations match well with the simulated results (less than $1 \%$ error), indicating that (11) and (12) are good approximations of the amplitude ratios.

It is further noticed that when $\Delta K / K<0$, the amplitude ratio of the out-of-phase mode has a much stronger dependency on the normalized stiffness perturbation than that of the in-phase mode; vice versa, when $\Delta K / K>0$, the in-phase mode amplitude ratio provides a more significant response. 


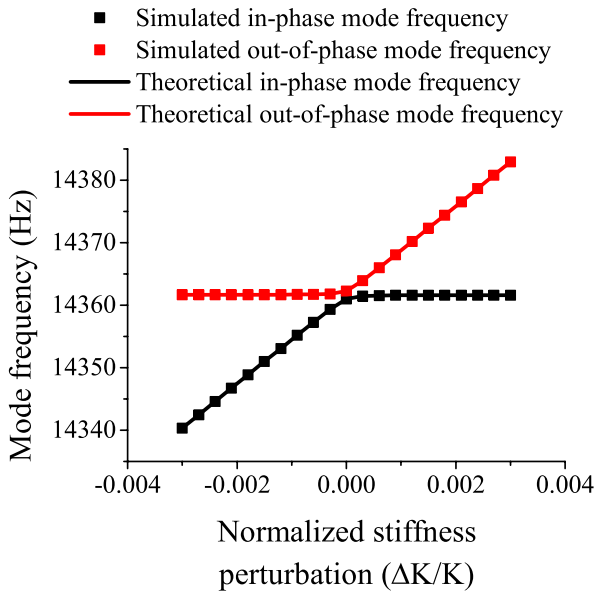

(a) Mode frequency with negligible damping

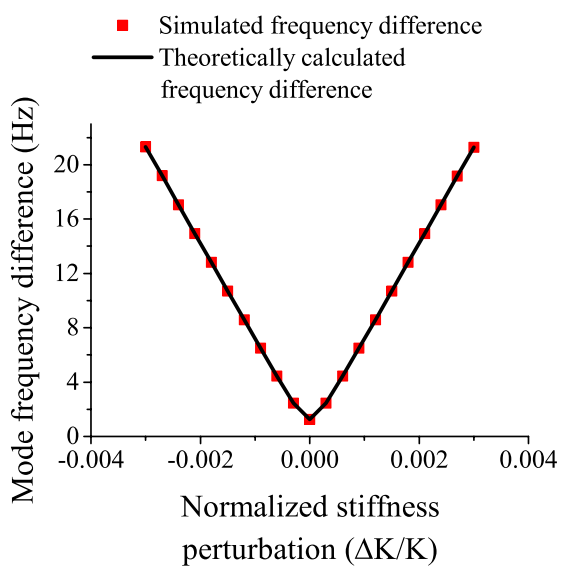

(b) Mode frequency difference with negligible damping

Fig. 3. Matlab calculation results with $\gamma=11325$ showing: a) the in-phase (black) and out-of-phase (red) mode frequencies as a function of a normalized stiffness perturbation $\Delta K / K$ comparing simulated and theoretically calculated values using (8) and (9); b) simulated and calculated mode frequency difference, $\Delta \omega=\omega_{\mathrm{op}}-\omega_{\mathrm{ip}}$. The theoretically calculated mode frequencies match well with the simulated values.

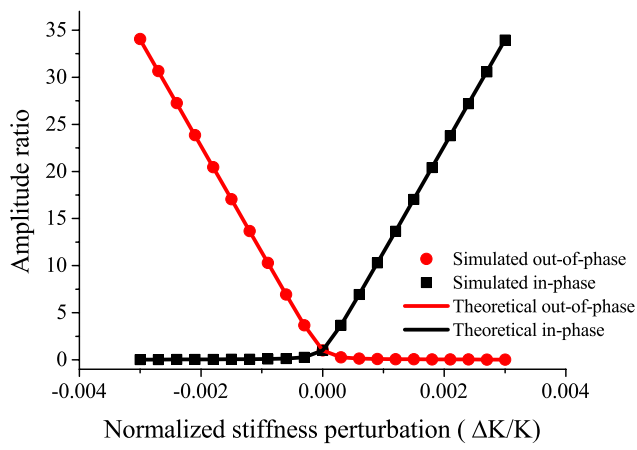

Fig. 4. Simulated (dots) with $\gamma=11325$ and theoretically estimated (lines) amplitude ratio (using (11) and (12)) for: in-phase (black) and out-of-phase (red) modes. The theoretically calculated amplitude ratios match well with simulated values.

Therefore, to obtain a higher sensitivity, for negative stiffness perturbations, the amplitude ratio of the out-of-phase mode should be chosen as the output; whereas for positive stiffness perturbations, the amplitude ratio of the in-phase mode is a better choice. Without loss of generality, the amplitude ratio of the out-of-phase mode for $\Delta K / K<0$ is chosen for the following analysis.

2) Linear Sensitivity to Stiffness Change: It can be seen from Fig. 4 that for negative stiffness perturbations, the amplitude ratio is approximately a linear function of stiffness perturbation. Assuming $|\gamma \Delta K / K|>10$, the amplitude ratio (12) can be linearized as [19]:

$$
\left|\frac{X_{1}\left(j \omega_{\mathrm{op}}\right)}{X_{3}\left(j \omega_{\mathrm{op}}\right)}\right|_{Q \rightarrow \infty} \approx-\frac{\gamma \Delta K}{K}
$$

Thus $-\gamma$ is the linear sensitivity of the device with respect to normalized stiffness perturbation $\Delta K / K$. Because of this, and its significance in the performance characteristics of the device as we shall see later, $\gamma$ is one of the most important parameters in our device. It is also worth noting from (10) that the linear sensitivity is determined by only the stiffness of the resonators and the coupling stiffness between them.

3) Nonlinearity of Amplitude Ratio Without Damping: Despite the fact that the amplitude ratio can be approximated as a linear function of the stiffness change, from (12) it can be seen that the amplitude ratio is a non-linear function of the normalized stiffness perturbation $\Delta K / K$. From the Taylor expansion, the nonlinearity error $\epsilon_{1}$ can be estimated as [19]:

$$
\epsilon_{1} \approx\left(\frac{K}{\gamma \Delta K}\right)^{2}
$$

It can be seen from (15) that the nonlinearity error $\epsilon_{1}$ increases with decreasing value of $(\gamma \Delta K / K)^{2}$. To demonstrate the influence of the nonlinearity, the same Matlab simulation as in the previous section is run, with varying $K_{c}$ in the model, thus resulting in different $K / K_{c}$ and $\gamma$ values. The $K_{c}$ values, along with the resulting $K / K_{c}$ and $\gamma$ values are listed in Table I. The results are presented in Fig. 5.

It can be seen from Fig. 5 that the amplitude ratio under the assumption of infinite quality factor can be approximated using the linearized scale function (14), with negligible nonlinearity errors (less than 1\%) for amplitude ratio larger than 10; however, the nonlinearity error increases as the stiffness perturbation approaches zero. Fig. $5 \mathrm{a}$ and $5 \mathrm{~b}$ show the results for two exemplary values of $\gamma$; it can be seen that for the same stiffness perturbation, a larger $\gamma$ results in better linearity. Fig. 5 also indicates that (15) can be regarded as an accurate estimation of the nonlinearity error.

\section{Analysis With Damping}

1) Dynamic Range: In reality, the quality factor will be a finite value due to damping in the system, hence leading to a finite bandwidth of each mode [26]. When one mode is in close vicinity to the other, the modes will interfere with each other, therefore mode aliasing occurs. The difficulty in identifying the mode of interest, i.e. the out-of-phase mode, 


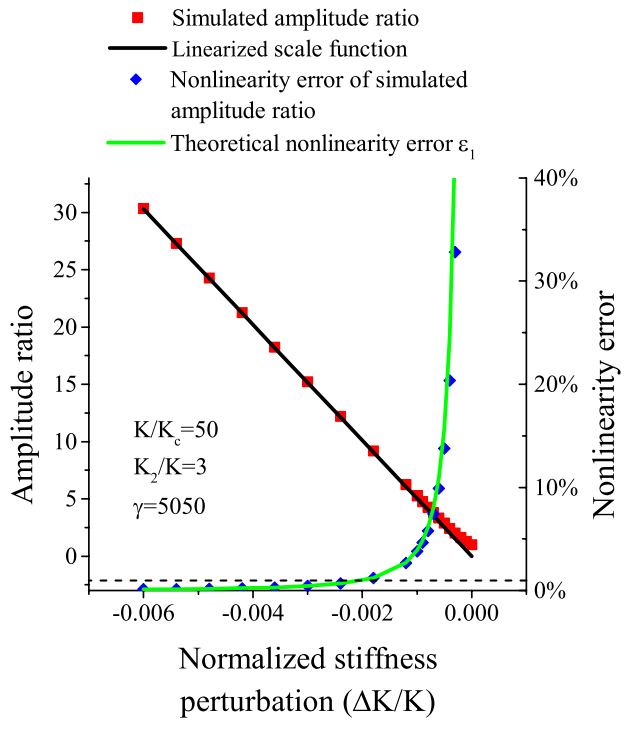

(a)

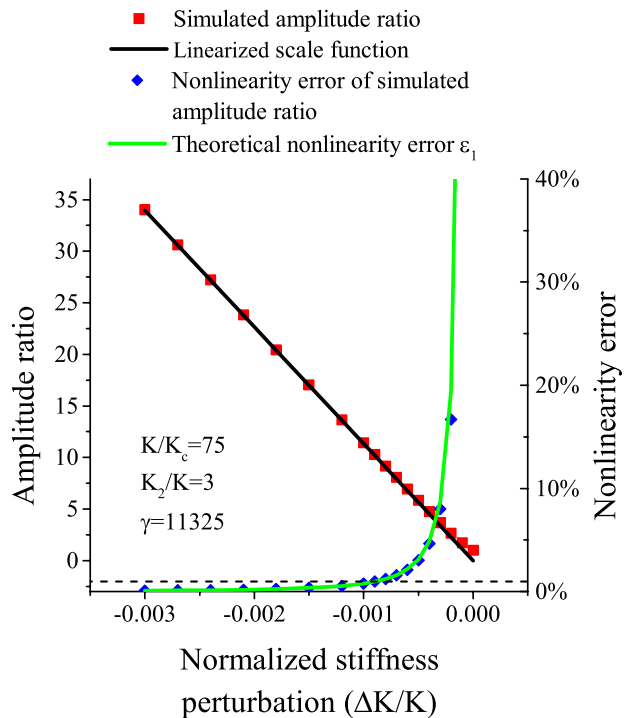

(b)

Fig. 5. Simulated amplitude ratios for a) $\gamma=5050$ and b) $\gamma=11325$ showing the linearized scale function given by (14), as well as the nonlinearity error. The nonlinearity error of the simulated amplitude ratio is calculated by comparing the simulated amplitude ratio to the linearized scale function, whereas the theoretical nonlinearity error is calculated using (15). The dashed lines at the bottom of the diagram mark a nonlinearity error of $1 \%$, which was regarded as negligible. The nonlinearity increases when the stiffness perturbation approaches zero. For the same stiffness perturbation, a larger $\gamma$ value results in a smaller nonlinearity error.

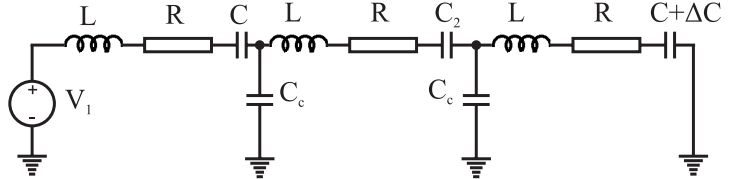

Fig. 6. Schematic of an equivalent electrical RLC model of a 3DoF resonator sensing device.

(in case of severe mode aliasing, modes could emerge) can lead to the sensor being unable to operate as intended [19]. To avoid severe mode aliasing, an anti-aliasing condition should be satisfied [19]:

$$
\Delta \omega>2 \times \Delta \omega_{3 \mathrm{~dB}}
$$

As shown in Fig. 3b, the frequency difference between the in-phase and out-of-phase mode is dependent on $\Delta K / K$. Therefore, for a given quality factor and $\gamma$ value, the antialiasing condition (16) sets a boundary for the maximum stiffness perturbation. Perturbation values beyond the boundary potentially result in severe mode aliasing effects.

To estimate this analytically, a simulation using an equivalent electrical RLC model [21] was run. Assuming the system is driven only by a force on resonator 1 as in previous sections, the schematic of the equivalent electrical RLC model is shown in Fig. 6. A quality factor of the modes of $Q=5000$ in vacuum was assumed; this is a conservative estimation compared to similar resonant devices [10], [27]. For this simulation, $C_{c}=19.07 \mathrm{fF}$; other values for the simulations are also listed in Table II.

As demonstrated in Fig. 7, (8) and (9) can still be regarded as accurate estimations of mode frequencies for $\Delta \omega \geq 2 \times \Delta \omega_{3 \mathrm{~dB}}$, with relative errors less than $25 \mathrm{ppm}$.
TABLE II

Values Used in the Simulation (With Damping)

\begin{tabular}{l|l|l}
\hline Component & Value & Mechanical model equivalent \\
\hline$L$ & $0.489 \mathrm{MH}$ & $M$ \\
\hline$C$ & $0.254 \mathrm{fF}$ & $K$ \\
\hline$C_{2}$ & $84.8 \mathrm{aF}$ & $K_{2} / K=3$ \\
\hline \multirow{2}{*}{$C_{C}$} & $19.07 \mathrm{fF}$ & $K / K_{c}=75, \gamma=11325$ \\
\cline { 2 - 3 } & $12.72 \mathrm{fF}$ & $K / K_{c}=50, \gamma=5050$ \\
\hline$R$ & $R=8.77 \mathrm{M} \Omega$ & $Q=5000$ \\
\hline
\end{tabular}

In addition, the 3-dB bandwidth, for a given finite quality factor $Q$, is given as:

$$
\Delta \omega_{3 \mathrm{~dB}}=\frac{1}{Q} \sqrt{\frac{K}{M}}
$$

From (8), (9), (16) and (17), for $\Delta K / K<0$ and $\gamma>Q / 2$, we can derive that the following condition should be satisfied:

$$
\frac{\Delta K}{K}<-2 \sqrt{\left(\frac{2}{Q}\right)^{2}-\left(\frac{1}{\gamma}\right)^{2}}
$$

Furthermore, the noise floor of the device and the interface circuitry sets the minimum detectable amplitude of the resonators; consequently a limitation of the maximum measurable amplitude ratio can be introduced. Thus, the lower boundary of the dynamic range of the device is determined as:

$$
\frac{\Delta K}{K}>-\frac{\text { Max Amplitude Ratio }}{\gamma}
$$

Therefore, it can be seen that the value of $\gamma$ should not be arbitrarily large due to its influence on the dynamic range of the sensor. 


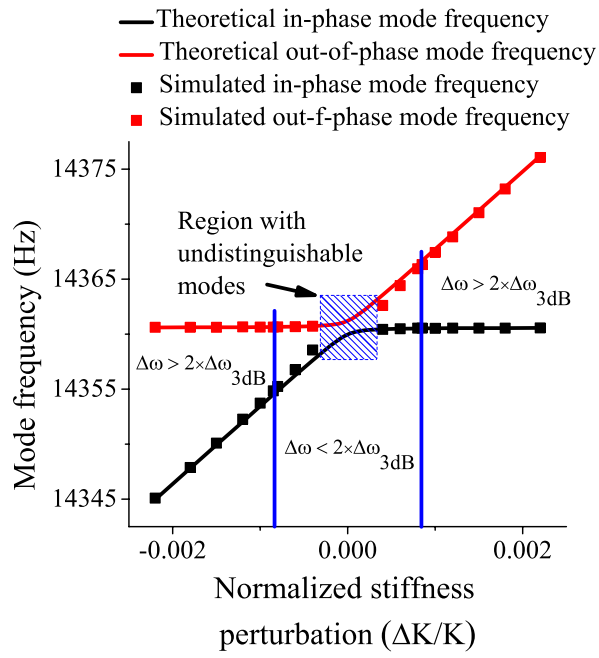

(a) Mode frequency with damping

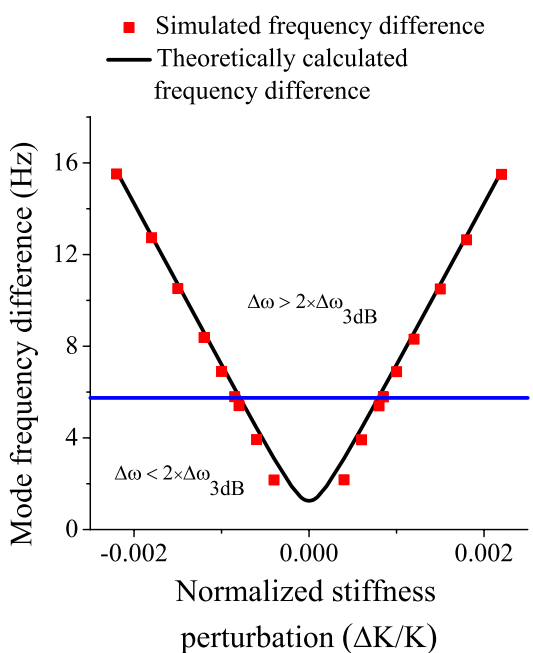

(b) Mode frequency difference with damping

Fig. 7. Simulation results with $\gamma=11325$ showing the in-phase and out-of-phase mode frequencies as a function of normalized stiffness: a) simulated with a quality factor of 5000 , and theoretically calculated mode frequencies using (8) and (9); b) simulated and calculated mode frequency difference, $\Delta \omega=\omega_{\text {op }}-\omega_{\text {ip }}$. The blue lines in both figures mark the boundary of $\Delta \omega=2 \times \Delta \omega_{3 \mathrm{~dB}}$. Regions where the anti-aliasing condition is satisfied are marked in both figures. The blue shaded area in a) shows the region where mode aliasing is so severe that the in-phase and out-of-phase modes cannot be differentiated. The theoretically calculated mode frequencies match well with simulated values, however, the error tends to grow when the frequency difference decreases in value.

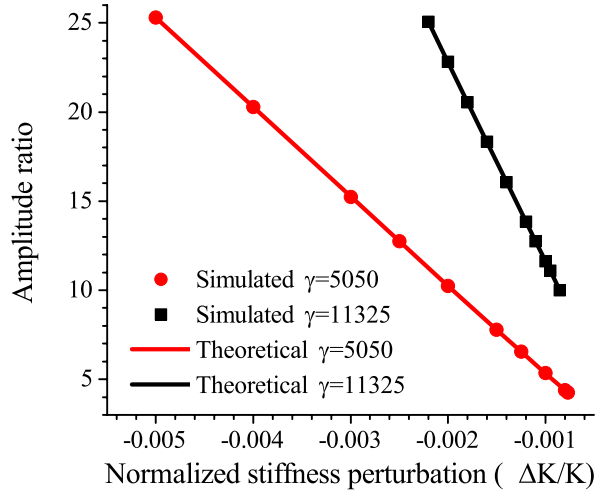

Fig. 8. Simulated (dots) and theoretically estimated (lines) amplitude ratio using (20) with $Q=5000$ but different coupling conditions and $\gamma$ values: $K / K_{c}=50, \gamma=5050$ (red); and $K / K_{c}=75, \gamma=11325$ (black). The simulated results matched well with theoretical predictions.

2) Amplitude Ratio Analysis With Damping: Within the dynamic range, the mode frequencies with finite damping can still be approximated by (8) and (9) if the condition of (7) is fulfilled. Hence, the amplitude ratio of the out-of-phase mode becomes:

$$
\left|\frac{X_{1}\left(j \omega_{\mathrm{op}}\right)}{X_{3}\left(j \omega_{\mathrm{op}}\right)}\right| \approx\left|-\frac{\sqrt{\gamma^{2}(\Delta K / K)^{2}+4}-\gamma(\Delta K / K)}{2}+j \frac{\gamma}{Q}\right|
$$

where $Q$ is the quality factor of the out-of-phase mode.

To verify (20), the same simulation was run using the values listed in Table II. The stiffness perturbations are chosen so that they are within the theoretical dynamic range, with the condition of (16) fulfilled.

It can be seen from Fig. 8 that the simulated results agree well with (20). Therefore, (20) can be considered as an accurate estimation of amplitude ratio with damping within the dynamic range.

3) Nonlinearity Analysis With Damping: Under the assumption that the nonlinear effects of the undamped and damped cases are uncoupled, the total nonlinearity error $\epsilon$ can be approximated as [19]:

$$
\epsilon \approx \epsilon_{1}+\epsilon_{2}=\left(\frac{K}{\gamma \Delta K}\right)^{2}+\frac{1}{2}\left(\frac{1}{Q} \frac{K}{\Delta K}\right)^{2}
$$

where $\epsilon_{1}$ is the nonlinearity term given by (15) and $\epsilon_{2}$ is a second nonlinearity term introduced by damping.

Simulated nonlinearity values in [19] showed good agreement to the theoretical values; however, it was not verified through measurement results. The measured nonlinear results will be compared to theoretical results in Section V-E.

\section{Theoretical Comparison to Other Resonant \\ Sensors Based on Stiffness Change}

From the previous analysis, the linear sensitivity of the 3 DoF resonant sensor, for negative $\Delta K / K$, is:

$$
\begin{aligned}
\mathcal{S}_{3 D o F} & =\frac{\partial(\text { Amplitude ratio })}{\partial(\Delta K / K)}=-\gamma \\
& =-\frac{K\left(K_{2}-K+K_{c}\right)}{K_{c}^{2}}
\end{aligned}
$$

In the same way as in [9] and [10], we can compare the amplitude ratio sensitivity of a $3 \mathrm{DoF}$ device to the resonant frequency sensitivity of a conventional resonant sensor (e.g. 1DoF) device. The sensitivity to normalized stiffness change of a 1 DoF resonant sensor with frequency shift output is given by (23) [28].

$$
\mathcal{S}_{1 D o F}=\frac{\partial(\text { Relative frequency shift })}{\partial(\Delta K / K)}=\frac{1}{2}
$$


(a)

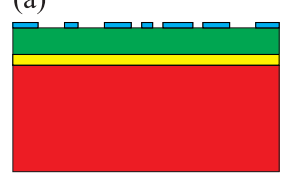

(b)
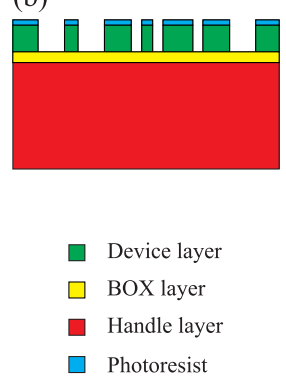

Fig. 9. The process flow of the single mask SOI process: a) deposition and patterning of photoresist, b) DRIE etching, c) overetching, d) photoresist removal and dicing, e) HF solution release.

With the assumptions stated in (7), the value for $\gamma$ is at least 100 , thus the improvement in sensitivity is at least two orders of magnitude.

For a $2 \mathrm{DoF}$ resonator sensor for stiffness change sensing applications, the sensitivity to a stiffness change can be approximated by [10]:

$$
\mathcal{S}_{2 D o F}=\frac{\partial(\text { Eigenstates shift })}{\partial(\Delta K / K)} \approx \frac{K}{4 K_{c}}
$$

Given identical $K$ and $K_{c}$ values, and with the assumption stated in (7), comparing (22) and (24), it is noticed that the sensitivity of a $3 \mathrm{DoF}$ resonator sensor is improved by $4\left(K_{2}-K+K_{c}\right) / K_{c}$, which is found to be at least 40 times.

\section{DEVICE DESCRIPTION}

\section{A. Fabrication Process}

To demonstrate the concept, a 3DoF resonator device was fabricated using a single mask silicon on insulator (SOI) process with a structural layer of $30 \mu \mathrm{m}$ thickness. The process flow is briefly summarized here (a more detailed description is provided in [29]), it comprised the following main steps:

1) Spin coating of photoresist on the front side of the SOI wafer, and patterning of the photoresist using a photomask, Fig. 9a.

2) Deep reactive ion etch (DRIE) to define the device layer structure, Fig. 9b,

3) Overetch step by DRIE, utilizing the notching effect [30], [31], Fig. 9c.

The notching only occurred when the trenches were narrower than a critical width, which was experimentally determined to be $16 \mu \mathrm{m}$. The bottom of all trenches narrower than $16 \mu \mathrm{m}$, including those between comb fingers, parallel plates and the majority of proof masses (due to release holes), were thus deliberately overetched and released. Structures with a larger area, such as the proof masses, were not completely released during this step; a small part on the edge of the proof masses was intentionally designed so that the proof masses and suspension beams were protected from shocks occurring during the dicing step.

To avoid the suspension beams from being overetched, the beams were placed well apart, much further away than the critical gap width of $16 \mu \mathrm{m}$, from any other structures. Therefore, negligible notching occurred at the bottom of these beams, and thus were not released in this step.

Due to the overetch step, stiction of the proof mass and other structures to the handle wafer was avoided during a final wet release step removing the buried oxide (BOX) layer [32], [33].

4) Removing the photoresist, followed by dicing, Fig. 9d. The BOX layer is retained in this step, so the fragile resonators structures will not be damaged during the dicing process.

5) Wet etching using HF solution to release the moving structures, including suspension beams and the edge of the proof masses, Fig. 9e.

\section{B. Device Design}

The designed system as shown in Fig. 1 consists of three resonators, coupled to its neighboring resonator through electrostatic springs [34]. To realize the electrostatic spring between the resonators, each resonator is placed next to its neighbor with a nominal air gap of $4.5 \mu \mathrm{m}$ in between. When a voltage difference is applied across the air gap between the resonators, an attractive electrostatic force is generated. If the displacement is small compared to the air gap, the electrostatic force is a linear function of the displacement, resulting in a behavior similar to a linear mechanical spring. If $\varepsilon_{0}, A, V, d$ are the permittivity of vacuum, cross-sectional area of the parallel plates, voltage difference and the gap between two proof masses, respectively, assuming a displacement much smaller than the air gap, and neglecting nonlinear terms, the stiffness of the electrostatic springs $K_{c}$ can be approximated by [34]:

$$
K_{c} \approx-\frac{\varepsilon_{0} A V^{2}}{d^{3}}
$$

A larger air gap $d$ gives a smaller nonlinearity for a given displacement [35], thus a larger air gap is preferred for increasing the displacement range for linear electrostatic spring. However, a larger gap also means that a higher voltage is required to obtain the same coupling strength. For a gap of $4.5 \mu \mathrm{m}$ chosen here, the resulting linear coupling spring constant $K_{c}$ ranges from 0.69 to $1.24 \mathrm{~N} / \mathrm{m}$ with the coupling voltage varying from $30-45 \mathrm{~V}$.

For each resonator, four suspension beams were used so that out of plane tilting is minimized. The beams form the suspension system of each resonator and can be treated as linear mechanical springs for small deflections. The suspension beams have one end fixed, while the other end moves perpendicular with respect to the beam length. Given the displacement functions along the axis of the beam for these boundary conditions [36], and with four beams in parallel for 
each resonator, the equivalent mechanical stiffness, $K_{m}$ (where $m$ denotes "mechanical") of each resonator can be derived as:

$$
K_{m}=4 \times \frac{E w^{3} t}{L^{3}}
$$

where $E, w, t, L$ are Young's modulus, width, thickness and length of each supporting beam, respectively.

A low mechanical stiffness of the resonator was desired in our design, as a smaller $K_{m}$ gives a lower resonant frequency (in the $\mathrm{kHz}$ range), simplifying the interface circuitry while retaining high sensitivity. The smallest beam width achievable with an acceptable yield in the fabrication process used was $5 \mu \mathrm{m}$, the minimum aspect ratio was $1: 70$, resulting in a beam length of $350 \mu \mathrm{m}$. The width of the suspension beams of the middle resonator, should be wider, resulting in a larger $K_{2}$, hence a larger $\gamma$ and thus higher sensitivity. However, as mentioned in the previous section, a too large $\gamma$ can limit the dynamic range of the device. A width of $7.5 \mu \mathrm{m}$ for the suspension beams of resonator 2 was regarded as adequate.

Relatively large proof masses were used to obtain low resonant frequencies (in the $\mathrm{kHz}$ range), and to make the proof masses less prone to random fabrication variations, as an important assumption is that all masses are equal. The dimensions of the proof masses of $360 \times 360 \mu \mathrm{m}^{2}$ were considered to be sufficient, resulting in a resonant frequency of approximately $15 \mathrm{kHz}$ (calculated using $E=169 \mathrm{GPa}$ and $\left.\rho=2.33 \mathrm{~g} / \mathrm{cm}^{3}\right)$.

An AC voltage, with a small amplitude compared to the fixed bias voltage on resonators, was applied to the electrode next to the left resonator. This created an alternating electrostatic force on resonator 1 , driving the $3 \mathrm{DoF}$ resonator sensor with only one actuation force. An actuation gap of $4.5 \mu \mathrm{m}$ was chosen to avoid significant nonlinear actuation force for a given displacement [37] while ensuring sufficient force to drive the resonators.

To sense the motions of resonators 1 and 3 , two sets of comb fingers were attached to the resonators on either side. Differential capacitive sensing was realized through the arrangements of the comb fingers. The length of the comb fingers were $90 \mu \mathrm{m}$; this ensures that the comb fingers will not vibrate. The overlap length was designed to be $70 \mu \mathrm{m}$ to get sufficient current to be measured with a standard transimpedance amplifier (TIA).

The design values are summarized in Table III.

\section{Perturbation Design}

When DC voltages were applied to resonators 1 and 3, electrostatic springs were also created between the resonators and the driving electrodes, as well as the sensing electrodes. Neglecting the higher order terms of the electrical spring stiffness, the effective stiffness of the outer resonator can be approximated as:

$K_{\mathrm{eff}}=K_{m}+K_{e} \approx \frac{4 E w^{3} t}{L^{3}}-\frac{\varepsilon_{0}\left(A V_{e}^{2}+6 \times A_{\mathrm{cf}} V_{c f}^{2}\right)}{d^{3}}$

where $K_{m}$ is the mechanical stiffness of the suspension beams, $K_{e}$ is the electrical spring stiffness arising when a bias voltage
TABLE III

Dimensions OF THE DEVICE

\begin{tabular}{l|l|l}
\hline Parameter & Design Value & Unit \\
\hline $\begin{array}{l}\text { Device layer thickness } \\
\text { Thickness of dry etch released area }(t)\end{array}$ & 30 & $\mu \mathrm{m}$ \\
\hline $\begin{array}{l}\text { Suspension beam lengths } \\
\text { (resonator 1, 2 and 3) }(L)\end{array}$ & 350 & $\mu \mathrm{m}$ \\
\hline $\begin{array}{l}\text { Suspension beam width } \\
\text { (resonator 1 and 3) }(w)\end{array}$ & 5 & $\mu \mathrm{m}$ \\
\hline $\begin{array}{l}\text { Suspension beam width } \\
\text { (resonator 2) }\left(w_{2}\right)\end{array}$ & 7.5 & $\mu \mathrm{m}$ \\
\hline $\begin{array}{l}\text { Gaps between resonators, between resonator } \\
\text { and electrodes, between comb fingers }(d)\end{array}$ & 4.5 & $\mu \mathrm{m}$ \\
\hline $\begin{array}{l}\text { Proof mass } \\
\text { Proportion of release holes in } \\
\text { a proof mass }\end{array}$ & $360 \times 360$ & $(\mu \mathrm{m})^{2}$ \\
\hline \begin{tabular}{l} 
Comb finger overlap \\
\hline $\begin{array}{l}\text { Cross-sectional area of } \\
\text { the parallel plates }(A)\end{array}$
\end{tabular} & 28.39 & $\%$ \\
\hline $\begin{array}{l}\text { Cross-sectional area of } \\
\text { the comb finger overlap }\left(A_{c f}\right)\end{array}$ & $70 \times 22$ & $(\mu \mathrm{m})^{2}$ \\
\hline
\end{tabular}

is applied on the resonator proof mass, $A_{\mathrm{cf}}$ is the crosssectional area of the comb finger overlap, $V_{e}$ is the voltage difference between resonator proof mass and neighboring electrode, and $V_{c f}$ is the voltage difference between the resonator proof mass and stationary comb fingers.

It is noticed from (27) that by altering the voltage on the electrode on the right, hence changing $V_{e}$, we can modify the effective stiffness of the resonator. Therefore, we are able to perturb the coupled resonator system with a stiffness change by altering $V_{e}$. Neglecting higher order terms, the perturbation in stiffness can be approximated as:

$$
\Delta K \approx-\frac{\varepsilon_{0} A}{d^{3}}\left[\left(V_{e}+\Delta V_{e}\right)^{2}-V_{e}^{2}\right]
$$

It should be pointed out that in presence of the perturbation voltage, the proof mass moves out of its rest position due to the electrostatic force, resulting in a small change in capacitive gap $d$. However, due to the relatively small perturbation voltages (less than $12 \mathrm{~V}$ ) applied in the experiments, the calculated resultant displacement was below $40 \mathrm{~nm}$, which was less than $1 \%$ compared to the designed air gap of $4.5 \mu \mathrm{m}$. Therefore (28) serves as a good first order approximation of the introduced stiffness perturbations.

Random fabrication variations in dimensions led to an intrinsic imbalance in the system. Because of the proof masses design, the mass variations were supposed to be negligible compared to the proof masses, therefore ignored in the analysis; only the effect of variations in stiffness is considered. The variations in dimensions led to an offset in stiffness; neglecting higher order terms, the normalized stiffness perturbation is deduced as:

$$
\frac{\Delta K}{K} \approx-\frac{\varepsilon_{0} A}{d^{3}}\left[\left(V_{e}+\Delta V_{e}\right)^{2}-V_{e}^{2}\right] / K_{\text {eff }}-\text { Offset }
$$

where $K_{\text {eff }}$ is the effective stiffness of the resonator given by (27). 


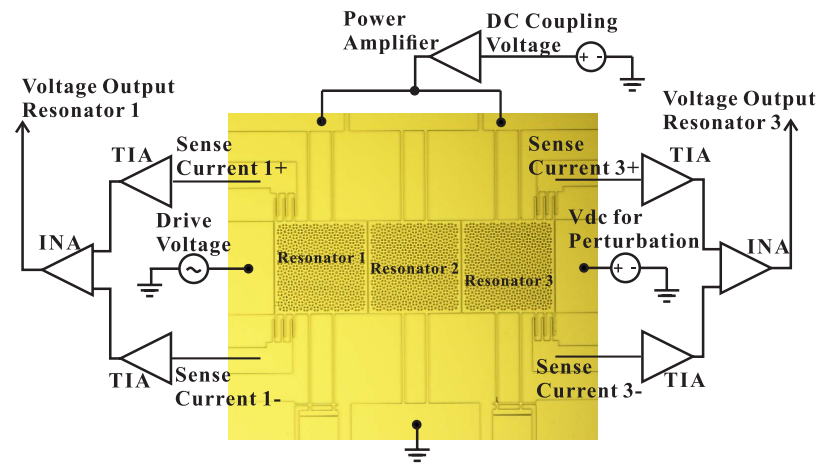

Fig. 10. Test configuration of the prototype $3 \mathrm{DoF}$ resonator sensing device along with the optical image of the $3 \mathrm{DoF}$ sensing device tested.

\section{EXPERIMENTAL METHODOLOGY}

\section{A. Device Configuration}

A prototype $3 \mathrm{DoF}$ resonator sensor was fabricated and configured as shown in Fig. 10 for characterization. The same DC voltage was applied to both proof masses of resonators 1 and 3, to ensure identical coupling, thus $K_{c 1}=K_{c 2}$.

An AC drive voltage was applied to the actuation electrodes on the left, so only $F_{1}$ was applied to the system, thus satisfying $F_{2}=F_{3}=0$.

A DC voltage was applied to the driving electrodes on the right. This created a DC voltage change in $V_{e}$, equivalent to the $\Delta V_{e}$ term in (28).

Differential sense currents were obtained from the comb fingers dedicated for motion sensing, and then fed to the interface circuitry for further processing.

\section{B. Electrical Test Set-Up}

To electrically test the chip, the chip was mounted on a chip carrier and wire bonded to the contacts. The chip carrier was then inserted into a socket on a printed circuit board. The circuit board was placed into a customized vacuum chamber with electrical feedthroughs. The ambient pressure was $20 \mu$ Torr ensuring minimum air damping loss, so a high quality factor could be obtained.

In four different sets of experiments, different coupling voltages were used. As discussed in section II-C.1, a too large $\gamma$ is not desired as it can limit the dynamic range of the device. To ensure the assumption in (7) is valid, the coupling voltage was set to be $30 \mathrm{~V}, 34.5 \mathrm{~V}, 40 \mathrm{~V}$ and $45 \mathrm{~V}$, respectively. The $K / K_{c}$ and $\gamma$ values were calculated using the design parameters and (10), and are listed in Table IV.

To drive the resonators, a sinusoidal AC voltage with adjustable frequency was generated from the signal generator. The peak to peak value was set to be $15 \mathrm{mV}$ to drive the resonators in the linear region. A real-time measurement method was employed in this work to measure the motion of resonators 1 and 3. Motional currents were amplified by standard TIAs (AD8065, Analog Devices Inc) and subsequent instrumentation amplifiers (INAs) (AD8421, Analog Devices Inc), and measured by a two-channel oscilloscope (DSO6032A from Agilent Technologies). The experimental method is described in detail elsewhere [19].
TABLE IV

CALCUlated AND EXPERIMENTALly EXTRACTED VAlues FOR DIFFERENT COUPLING VOLTAGES

\begin{tabular}{l|l|l|l|l}
\hline $\begin{array}{l}\text { Coupling } \\
\text { voltage }(\mathrm{V})\end{array}$ & $\begin{array}{l}\text { Calculated } \\
K / K_{c}\end{array}$ & $\begin{array}{l}\text { Calculated } \\
\gamma\end{array}$ & $\begin{array}{l}\text { Extracted } \\
\gamma\end{array}$ & $\begin{array}{l}\text { Extracted offset } \\
\text { of } \Delta K / K\end{array}$ \\
\hline 30 & 83.5 & 17073 & 13558 & $3.8 \times 10^{-3}$ \\
34.5 & 62.4 & 9656 & 9118 & $2.6 \times 10^{-3}$ \\
40 & 45.7 & 5250 & 5534 & $9.1 \times 10^{-4}$ \\
45 & 35.7 & 3259 & 3538 & $-5.7 \times 10^{-4}$ \\
\hline
\end{tabular}

\section{RESUlts AND Discussion}

\section{A. $\gamma$ Value and Offset Extraction}

Before proceeding to illustrate of the functionality of the sensor, it is important to quantify the $\gamma$ values, since the performance of the sensor depends critically on this parameter. In addition, the offset values in stiffness perturbations $\Delta K / K$ were characterized due to its importance in analyzing the measurement results.

To approximate the $\gamma$ and offset values from the measurement data, the linearized scale function (14) was utilized. The reason for this is that the $\gamma$ values were different to the designed values due to the fabrication tolerances, as will be discussed in Section V-D. Both the $\gamma$ and offset values were estimated by fitting the measured data for amplitude ratios larger than 20 to a linear function. The slope of the line was expected to be a good approximation of $-\gamma$ and the intersection of the line to the horizontal axis was regarded as a good estimation of the offset value. The estimated $\gamma$ values and offset values are listed in Table IV. The $\Delta K / K$ values in the following sections were deduced using (29) including the experimentally estimated offset values.

\section{B. Frequency Response}

By using the experimental set up and method described in the previous section, we measured the frequency response of the 3DoF resonator sensing device as shown in Fig. 11. For $4.85 \mathrm{~V}$ perturbation voltage, the corresponding stiffness perturbation $\Delta K / K$ was calculated as $-0.23 \times 10^{-3}$ according to (29). Two modes were close to each other, thus a strong mode aliasing effect was observed, as shown in Fig. 11a. When we changed the perturbation voltage to $4.15 \mathrm{~V}$, hence decreasing the value of $\Delta K / K$ to $-0.69 \times 10^{-3}$, the frequency difference between the in-phase and out-of-phase modes became larger, as predicted by [19], the mode aliasing effect became weaker, and the in-phase and out-of-phase modes could be identified, as shown in Fig. $11 \mathrm{~b}$.

The frequencies of the in-phase and the out-of-phase modes were found to be $14925.42 \mathrm{~Hz}$ and $14930.41 \mathrm{~Hz}$, respectively; while the calculated corresponding mode frequencies were $14414.76 \mathrm{~Hz}$ and $14419.90 \mathrm{~Hz}$, respectively; hence there is good agreement between measured and theoretical values.

The measured $3 \mathrm{~dB}$ bandwidth of the out-of-phase mode was $2.4 \mathrm{~Hz}$; from this, the quality factor could be calculated as approximately 6221, which was sufficiently close to the assumed quality factor of 5000 in the analytical derivations. 


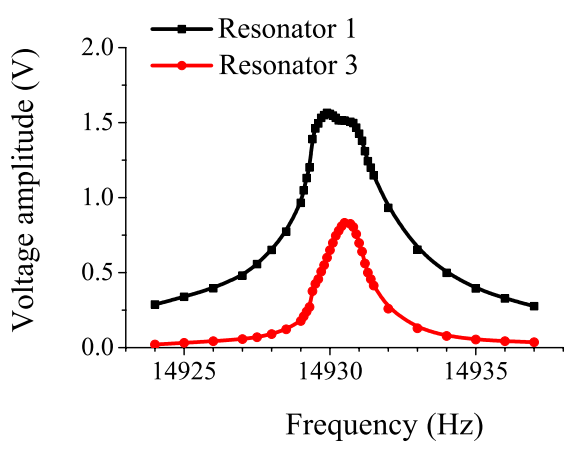

(a) Strong mode aliasing

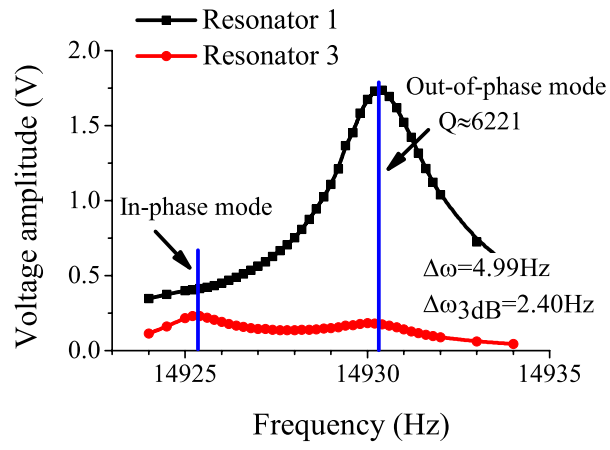

(b) Weak mode aliasing

Fig. 11. Measured frequency response of resonators 1 and 3, with $30 \mathrm{~V}$ coupling voltage, corresponding to $K / K_{C}=83.5$ and $\gamma=17073$. a) For a perturbation voltage of $4.85 \mathrm{~V}$, equivalent to $\Delta K / K=-0.23 \times 10^{-3}$, strong mode aliasing occurs and therefore the out-of-phase mode was difficult to identify; b) for a perturbation voltage of $4.15 \mathrm{~V}$, equivalent to $\Delta K / K=-0.69 \times 10^{-3}$, weak mode aliasing occurred and the out-of-phase mode could be identified.

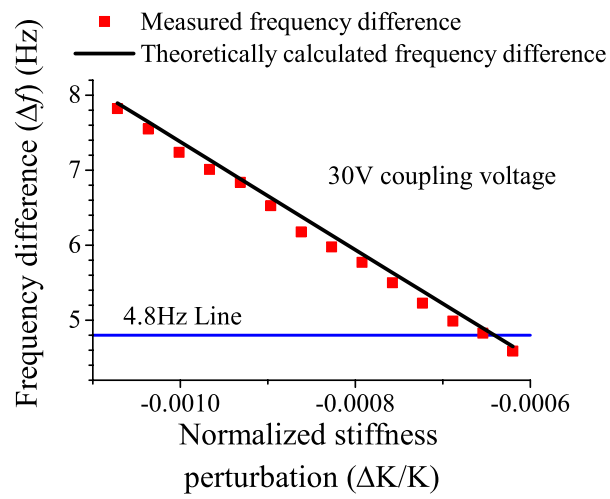

(a) Mode frequency difference with $30 \mathrm{~V}$ coupling voltage

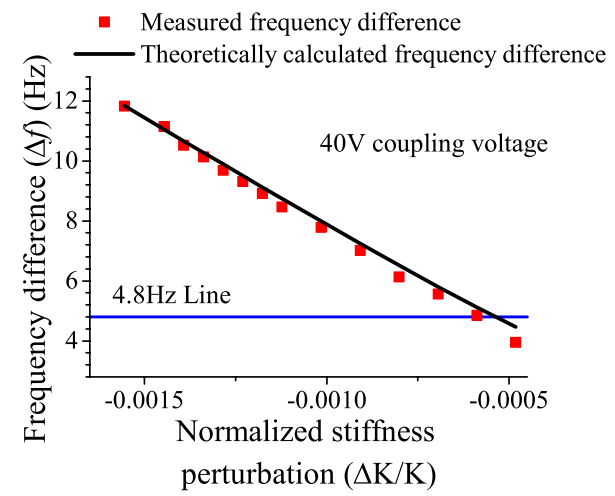

(c) Mode frequency difference with $40 \mathrm{~V}$ coupling voltage

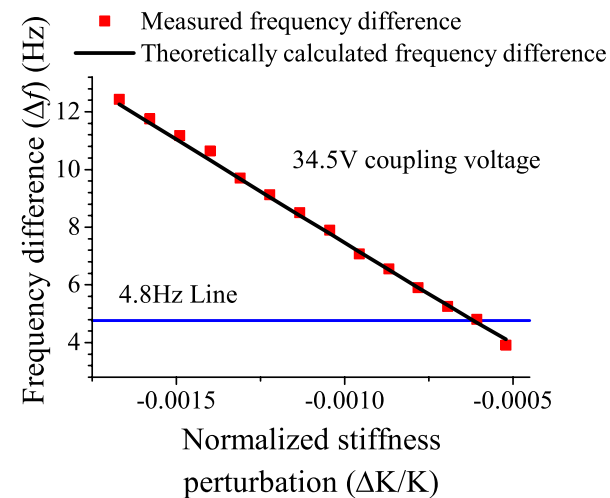

(b) Mode frequency difference with $34.5 \mathrm{~V}$ coupling voltage

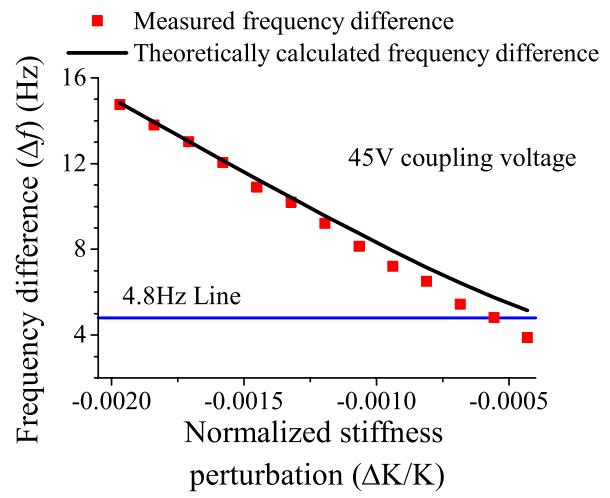

(d) Mode frequency difference with $45 \mathrm{~V}$ coupling voltage

Fig. 12. Measured and theoretically calculated frequency differences with different coupling voltages: a) $30 \mathrm{~V}$ coupling voltage, b) $34.5 \mathrm{~V}$ coupling voltage, c) $40 \mathrm{~V}$ coupling voltage, d) $45 \mathrm{~V}$ coupling voltage. The measured frequency differences matched well with the theoretical calculations.

\section{Dynamic Range}

To avoid strong mode aliasing as shown in Fig. 11a, the anti-aliasing condition given by (16) should be satisfied. For the bandwidth measured in the experiment, the minimum frequency difference required was $2 \times \Delta \omega_{3 \mathrm{~dB}}=4.8 \mathrm{~Hz}$.

Mode frequency measurements were carried out to find the boundary of the perturbation values to satisfy a minimum frequency difference of $4.8 \mathrm{~Hz}$. The frequency differences versus stiffness perturbations are plotted in Fig. 12, together with the theoretically calculated frequency differences using extracted $\gamma$ and offset values from the previous sections. The measured frequencies matched well with the theoretical calculations.

The lower boundary of the dynamic range was limited by the noise of the resonator and the associated circuitry interface, as discussed in section II-C1. In our experiment, for decreasing 
TABLE V

DyNAMIC RANGe IN TERMS OF NORMALIZEd CHANGE IN STIFFNESS

\begin{tabular}{l|l|l|l|l}
\hline $\begin{array}{l}\text { Coupling } \\
\text { voltage }(\mathrm{V})\end{array}$ & Extracted & $\begin{array}{l}\text { Lower boundary } \\
\Delta K / K\end{array}$ & $\begin{array}{l}\text { Upper boundary } \\
\Delta K / K\end{array}$ & $\begin{array}{l}\text { Dynamic range } \\
\Delta K / K\end{array}$ \\
\hline 30 & 13358 & $-3.78 \times 10^{-3}$ & $-0.654 \times 10^{-3}$ & $3.12 \times 10^{-3}$ \\
34.5 & 9118 & $-5.49 \times 10^{-3}$ & $-0.607 \times 10^{-3}$ & $4.89 \times 10^{-3}$ \\
40 & 5534 & $-9.05 \times 10^{-3}$ & $-0.589 \times 10^{-3}$ & $8.46 \times 10^{-3}$ \\
45 & 3538 & $-14.77 \times 10^{-3}$ & $-0.558 \times 10^{-3}$ & $14.2 \times 10^{-3}$ \\
\hline
\end{tabular}

perturbations, the amplitude of resonator 3 became smaller, consequently limiting the maximum amplitude ratio that could be detected. The measured output noise for resonator 3 without driving signal was approximately $3 \mathrm{mVrms}$, after 500 cycles of averages. The noise was attributed to the electrical and mechanical noise from the circuit and the MEMS device respectively [19]. According to the theory in [19] and [26], the output signal-to-noise ratio (SNR) is limited by the SNR of the resonator with smaller amplitudes. Therefore, to ensure an output SNR of at least $10 \mathrm{~dB}$, the minimum peak-peak amplitude of resonator 3 was $30 \mathrm{mV}$, resulting in maximum amplitude ratios between approximately 50 to 52 in four different measurement sets.

The boundaries of the dynamic range of the sensor, due to these limitations from the mode aliasing effect and the maximum amplitude ratio, for different coupling voltages are listed in Table V.

As demonstrated here, a higher $\gamma$ value narrowed the dynamic range of the sensor. This is because the lower limit of the dynamic range is inversely proportional to $\gamma$, as predicted by (19), with a given maximum obtainable amplitude ratio, as shown in the Table V. Moreover, as implied by (18), the upper boundary increases as $\gamma$ decreases, which confirms the trends seen in Table V. Combining these two observations, it can be concluded that the dynamic range decreases with increasing $\gamma$ values.

\section{Amplitude Ratio and Sensitivity Analysis}

The estimated theoretical amplitude ratios using extracted $\gamma$ values, measured quality factor and (20) are depicted in Fig. 13. From Fig. 13, it can be seen that the measured results matched well with the theoretical predictions, with a relative error smaller than $5 \%$ in all cases.

The measurement result also showed that a smaller coupling voltage led to a higher amplitude ratio for a given perturbation stiffness, thus a higher sensitivity. The linear sensitivity of the sensor, extracted from the measurement data, together with the sensitivity calculated using design values are listed in Table VI.

The discrepancies (relative error shown in Table VI) between measured and ideal sensitivity were attributed to fabrication variations, and due to the high sensitivity of the device, small parameter variations were also amplified. This should be improved in the future.

\section{E. Nonlinearity}

To calculate the total nonlinearity errors, the measured data was compared to the linearized scale function (14). The results are plotted in Fig. 14.

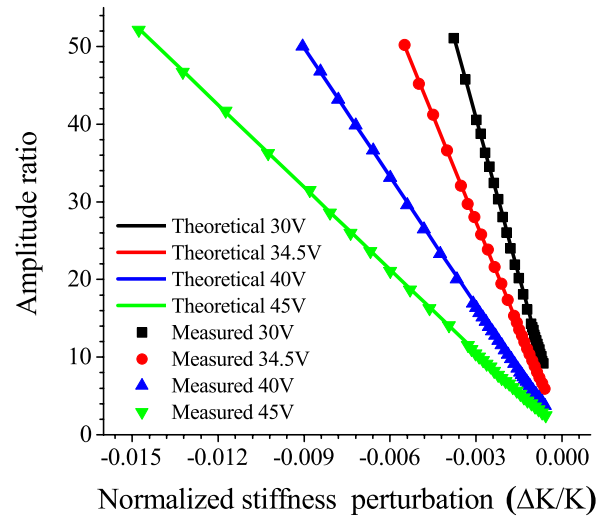

Fig. 13. Measured (dots) and theoretically estimated (lines) amplitude ratio using (20) with different coupling voltages: $30 \mathrm{~V}$ coupling voltage (black), $34.5 \mathrm{~V}$ coupling voltage (red), $40 \mathrm{~V}$ coupling voltage (blue), $45 \mathrm{~V}$ coupling voltage (green). The measurement results matched well with theoretical predictions.

TABLE VI

SENSITIVITY ANALYSIS

\begin{tabular}{l|l|l|l}
\hline $\begin{array}{l}\text { Coupling } \\
\text { voltage }(\mathrm{V})\end{array}$ & $\begin{array}{l}\text { Linear sensitivity } \\
\text { (measured data) }\end{array}$ & $\begin{array}{l}\text { Linear sensitivity } \\
\text { (design values) }\end{array}$ & $\begin{array}{l}\text { Relative error } \\
\text { of sensitivity }\end{array}$ \\
\hline 30 & -13558 & -17073 & $-20.59 \%$ \\
34.5 & -9118 & -9656 & $-5.57 \%$ \\
40 & -5534 & -5250 & $5.40 \%$ \\
45 & -3538 & -3259 & $8.56 \%$ \\
\hline
\end{tabular}

From Fig. 14, it is found that the theoretically estimated nonlinearity errors matched well with the measured errors. Also, as predicted by theory, the nonlinearity error tended to increase as the stiffness perturbation approached zero; and with decreasing $\gamma$ value, the maximum nonlinearity error in the dynamic range increased. Nonetheless, for a wide span of stiffness perturbations, the sensor provides good linearity, with a typical nonlinearity error smaller than $\pm 2 \%$.

\section{F. Discussion}

From the measurement results, we obtained the linear sensitivity of the sensor. Comparing the measured linear sensitivity to the state-of-the-art resonator sensors reported in the literature, a significant improvement in sensitivity is noticed. A brief comparison of the sensitivity is shown in Table VII.

It is seen from Table VII that the improvement in sensitivity was significant: compared to the conventional single resonator sensor with frequency shift output, the improvement was over four orders of magnitude; whereas the improvement 


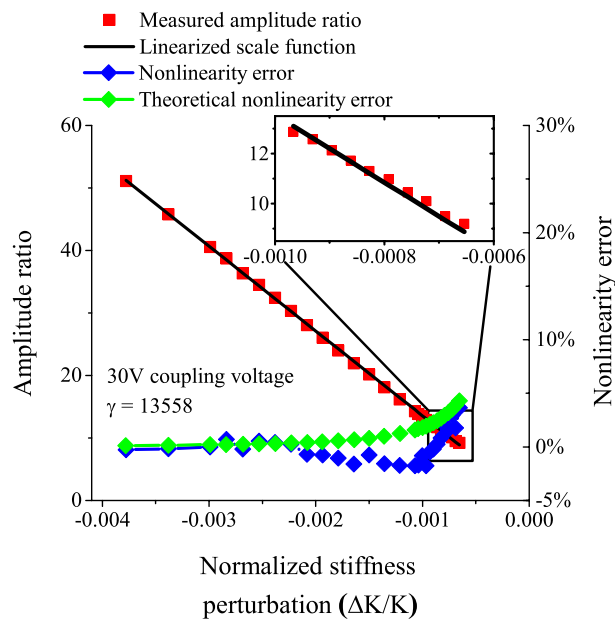

(a) Nonlinearity with $30 \mathrm{~V}$ coupling voltage

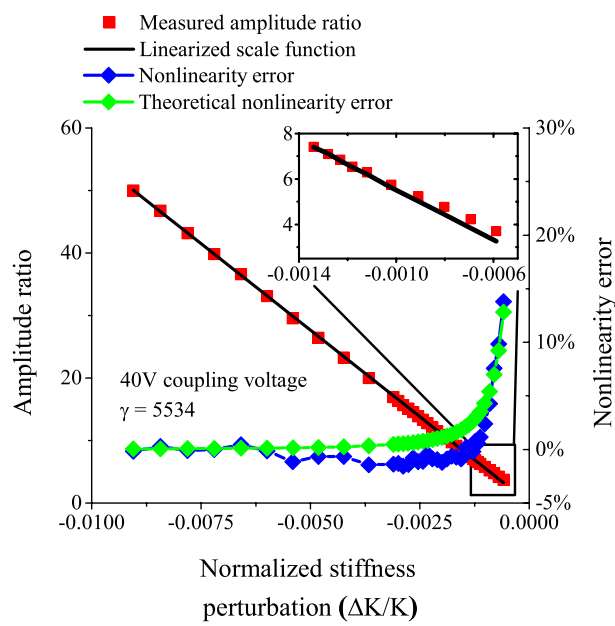

(c) Nonlinearity with $40 \mathrm{~V}$ coupling voltage

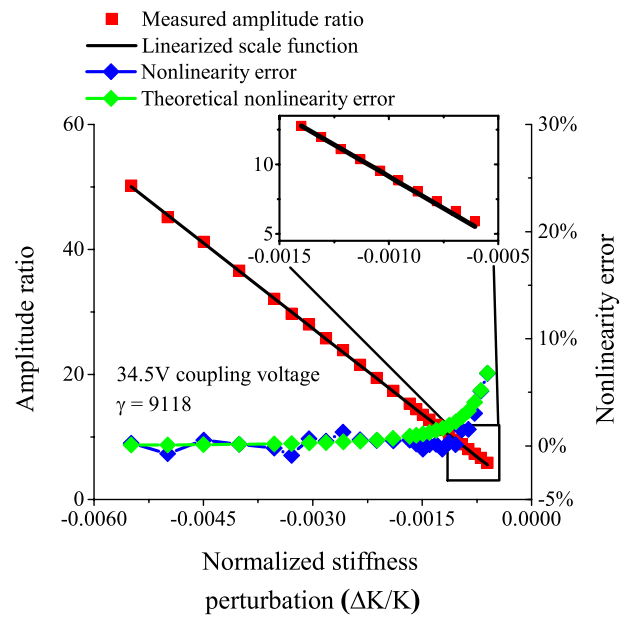

(b) Nonlinearity with $34.5 \mathrm{~V}$ coupling voltage

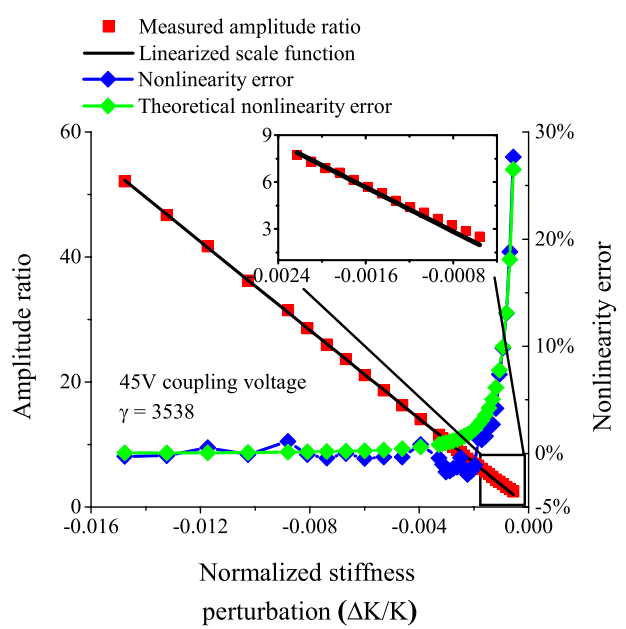

(d) Nonlinearity with $45 \mathrm{~V}$ coupling voltage

Fig. 14. Comparison of measured amplitude ratio to linearized scale function (14) with different coupling voltages: a) $30 \mathrm{~V}$ coupling voltage, b) $34.5 \mathrm{~V}$ coupling voltage, c) $40 \mathrm{~V}$ coupling voltage, d) $45 \mathrm{~V}$ coupling voltage. The nonlinearity error was calculated by comparing the measured amplitude ratio to linearized scale function given by (14) and displayed in blue. The theoretical nonlinearity error was calculated using, $\epsilon_{1}+\epsilon_{2}$ given by (21). The theoretically estimated nonlinearity errors matched well with measured errors with tolerable discrepancies, which was mainly due to the error in measurement and extraction of $\gamma$ and offset values.

TABLE VII

SENSITIVITy COMPARISON With THE StATE-OF-THE-ART RESONATOR SENSORS FOR STIFFNESS CHANGE

\begin{tabular}{l|l|l}
\hline Reference & Sensitivity & Type \\
\hline$[28]$ & 0.5 & 1DoF resonant sensor \\
{$[11]$} & $\sim 20$ & 2DoF resonant sensor \\
{$[13]$} & $\sim 241$ & 2DoF resonant sensor \\
Our work & $\sim 13558$ & 3DoF resonant sensor \\
\hline
\end{tabular}

in sensitivity compared to the $2 \mathrm{DoF}$ sensor utilizing mode localization was over 49 times.

It can be found from the measurement results that the dynamic range was not large (Table $\mathrm{V}$ ). This was due to a relatively high value of minimum detectable stiffness change, i.e. the upper boundary of the stiffness perturbation as shown in Table V. One way to enhance the dynamic range is to add a perturbation bias $\Delta K_{\text {bias }}<\Delta K_{\text {upper }}$ intentionally, and it was successfully used in [19] to improve the dynamic range.
For a coupling voltage of $30 \mathrm{~V}$, based on the results shown in Table $\mathrm{V}$, if the sensor was biased at the upper boundary, i.e. $\Delta K / K=-0.654 \times 10^{-3}$, the full range of the sensor would be $3.12 \times 10^{-3}$. We estimate the noise floor as the minimum step of $\Delta K / K$ in the experiment, which was approximately $3.4 \times 10^{-5}$. A conservative estimation of the dynamic range was therefore $39.1 \mathrm{~dB}$, which is acceptable for a prototype device.

The dynamic range can further be extended by decreasing the noise of the interface electronics, hence improving the limits of the maximum amplitude ratio that can be detected, as indicated by (19), consequently increasing the dynamic range of the sensor.

A property of the sensor is that the linearity of sensor is not trivial for a stiffness perturbation approaching zero, hence limiting the measurement range and increasing the burden of calibration in the meantime. However, as demonstrated from the theory and measurement results, increasing stiffness 
perturbation (negative) results in a smaller nonlinearity error. Hence, the linearity can be improved by adding a stiffness perturbation bias intentionally to a point above which nonlinearity error is tolerable for the intended applications.

\section{CONCLUSIONS}

In this paper, we have reported a $3 \mathrm{DoF}$ resonator sensor device for stiffness change sensing applications. The measured sensitivity of a prototype sensor represents an improvement by over 49 times compared to the state-of-the-art stiffness change sensors consisting of two weakly coupled resonators. Also in this paper, the dynamic range of this type of sensor is analyzed in detail for the first time. It is demonstrated by theory and experiment that there is a trade-off between the sensitivity and the dynamic range of the sensor. In the future, this constraint can be loosened by limiting the noise of both the sensor and interface circuit, while improving the quality factor of the resonator. One disadvantage of the current prototype is that it requires the user to adjust the drive signal frequency to find the resonant frequency, which is time consuming, thus limiting the use of the sensor for real time measuring applications. With a self-oscillating loop design, combined with an enhanced, as well as tunable sensitivity, the $3 \mathrm{DoF}$ resonator sensor can be a potentially attractive alternative to the more conventional resonator sensors.

\section{REFERENCES}

[1] M. A. Schmidt and R. T. Howe, "Silicon resonant microsensors," in Proc. 14th Auto. Mater. Conf., Ceram. Eng. Sci., 2008, vol. 8. nos. 9-10, pp. 1019-1034.

[2] H. A. C. Tilmans, M. Elwenspoek, and J. H. J. Fluitman, "Micro resonant force gauges," Sens. Actuators A, Phys., vol. 30, nos. 1-2, pp. 35-53, Jan. 1992.

[3] K. Jensen, K. Kim, and A. Zettl, "An atomic-resolution nanomechanical mass sensor," Nature Nanotechnol., vol. 3, no. 9, pp. 533-537, Sep. 2008.

[4] A. A. Seshia et al., "A vacuum packaged surface micromachined resonant accelerometer," J. Microelectromech. Syst., vol. 11, no. 6, pp. 784-793, Dec. 2002.

[5] R. G. Azevedo et al., "A SiC MEMS resonant strain sensor for harsh environment applications," IEEE Sensors J., vol. 7, no. 4, pp. 568-576, Apr. 2007.

[6] K. Petersen, F. Pourahmadi, J. Brown, P. Parsons, M. Skinner, and J. Tudor, "Resonant beam pressure sensor fabricated with silicon fusion bonding," in Int. Conf. Solid-State Sens. Actuators Dig. Tech. Papers (TRANSDUCERS), Jun. 1991, pp. 664-667.

[7] U. Dürig, J. K. Gimzewski, and D. W. Pohl, "Experimental observation of forces acting during scanning tunneling microscopy," Phys. Rev. Lett., vol. 57, pp. 2403-2406, Nov. 1986.

[8] T. R. Albrecht, P. Grütter, D. Horne, and D. Rugar, "Frequency modulation detection using high- $Q$ cantilevers for enhanced force microscope sensitivity," J. Appl. Phys., vol. 69, no. 2, p. 668, 1991.

[9] M. Spletzer, A. Raman, A. Q. Wu, X. Xu, and R. Reifenberger, "Ultrasensitive mass sensing using mode localization in coupled microcantilevers," Appl. Phys. Lett., vol. 88, no. 25, p. 254102, 2006.

[10] P. Thiruvenkatanathan, J. Yan, J. Woodhouse, and A. A. Seshia, "Enhancing parametric sensitivity in electrically coupled MEMS resonators," J. Microelectromech. Syst., vol. 18, no. 5, pp. 1077-1086, Oct. 2009.

[11] M. Manav, G. Reynen, M. Sharma, E. Cretu, and A. S. Phani, "Ultrasensitive resonant MEMS transducers with tuneable coupling," J. Micromech. Microeng., vol. 24, no. 5, p. 055005, 2014.

[12] P. W. Anderson, "Absence of diffusion in certain random lattices," Phys. Rev., vol. 109, no. 5, pp. 1492-1505, Mar. 1958.

[13] P. Thiruvenkatanathan, J. Yan, and A. Seshia, "Ultrasensitive modelocalized micromechanical electrometer," in Proc. IEEE Int. Freq. Control Symp. (FCS), Jun. 2010, pp. 91-96.
[14] P. Thiruvenkatanathan and A. A. Seshia, "Mode-localized displacement sensing," J. Microelectromech. Syst., vol. 21, no. 5, pp. 1016-1018, Oct. 2012.

[15] S. Hafizi-Moori and E. Cretu, "Weakly-coupled resonators in capacitive readout circuits," IEEE Trans. Circuits Syst. I, Reg. Papers, vol. 62, no. 2, pp. 337-346, Feb. 2015.

[16] P. Thiruvenkatanathan, J. Woodhouse, J. Yan, and A. A. Seshia, "Manipulating vibration energy confinement in electrically coupled microelectromechanical resonator arrays," J. Microelectromech. Syst., vol. 20, no. 1, pp. 157-164, Feb. 2011

[17] M. Spletzer, A. Raman, H. Sumali, and J. P. Sullivan, "Highly sensitive mass detection and identification using vibration localization in coupled microcantilever arrays," Appl. Phys. Lett., vol. 92, no. 11, p. 114102, 2008.

[18] J. A. Harley and T. W. Kenny, "A high-stiffness axial resonant probe for atomic force microscopy," J. Microelectromech. Syst., vol. 10, no. 3, pp. 434-441, Sep. 2001.

[19] C. Zhao, G. S. Wood, J. Xie, H. Chang, S. H. Pu, and M. Kraft, "A force sensor based on three weakly coupled resonators with ultrahigh sensitivity," Sens. Actuators A, Phys., vol. 232, pp. 151-162, Aug. 2015.

[20] W.-C. Li, Y. Lin, B. Kim, Z. Ren, and C. T.-C. Nguyen, "Quality factor enhancement in micromechanical resonators at cryogenic temperatures," in Proc. Int. Solid-State Sens., Actuators, Microsyst. Conf. (TRANSDUCERS), Jun. 2009, pp. 1445-1448.

[21] C. T.-C. Nguyen, "Frequency-selective MEMS for miniaturized lowpower communication devices," IEEE Trans. Microw. Theory Techn., vol. 47, no. 8, pp. 1486-1503, Aug. 1999.

[22] C. Pierre, "Mode localization and eigenvalue loci veering phenomena in disordered structures," J. Sound Vibrat., vol. 126, no. 3, pp. 485-502, 1988.

[23] C. Zhao, G. S. Wood, J. Xie, H. Chang, S. H. Pu, and M. Kraft, "Comparative study of different output metrics for a three weakly coupled resonator sensor," in Proc. 18th Int. Conf. Solid-State Sens., Actuators, Microsyst. (TRANSDUCERS), 2015, pp. 2196-2199.

[24] C. Zhao et al., "A sensor for stiffness change sensing based on three weakly coupled resonators with enhanced sensitivity," in Proc. IEEE 28th Int. Conf. Micro Electro Mech. Syst. (MEMS), Jan. 2015, pp. 881-884.

[25] P. Thiruvenkatanathan, "Mode-localized sensing in micromechanical resonator arrays," Ph.D. dissertation, Dept. Eng., Univ. Cambridge, Cambridge, U.K., 2010.

[26] P. Thiruvenkatanathan, J. Woodhouse, J. Yan, and A. A. Seshia, "Limits to mode-localized sensing using micro- and nanomechanical resonator arrays," J. Appl. Phys., vol. 109, no. 10, p. 104903, 2011.

[27] K. Wang and C. T.-C. Nguyen, "High-order micromechanical electronic filters," in Proc. IEEE 10th Аnnu. Int. Workshop Micro Electro Mech. Syst. (MEMS), Jan. 1997, pp. 25-30.

[28] F. J. Giessibl, "A direct method to calculate tip-sample forces from frequency shifts in frequency-modulation atomic force microscopy," Appl. Phys. Lett., vol. 78, no. 1, p. 123, 2001.

[29] J. B. Xie, Y. C. Hao, H. L. Chang, and W. Z. Yuan, "Single mask selective release process for complex SOI MEMS device," Key Eng. Mater, vols. 562-565, pp. 1116-1121, Jul. 2013.

[30] P. T. Docker, P. Kinnell, and M. C. L. Ward, "A dry single-step process for the manufacture of released MEMS structures," J. Micromech. Microeng., vol. 13, no. 5, p. 790, 2003.

[31] L. Haobing and F. Chollet, "Layout controlled one-step dry etch and release of MEMS using deep RIE on SOI wafer," J. Microelectromech. Syst., vol. 15, no. 3, pp. 541-547, Jun. 2006.

[32] I. Sari, I. Zeimpekis, and M. Kraft, "A dicing free SOI process for MEMS devices," Microelectron. Eng., vol. 95, pp. 121-129, Jul. 2012.

[33] O. Raccurt, F. Tardif, F. A. d'Avitaya, and T. Vareine, "Influence of liquid surface tension on stiction of SOI MEMS," J. Micromech. Microeng., vol. 14, no. 7, p. 1083, 2004.

[34] S. Pourkamali and F. Ayazi, "Electrically coupled MEMS bandpass filters: Part II. Without coupling element," Sens. Actuators A, Phys., vol. 122, no. 2, pp. 317-325, Aug. 2005.

[35] V. Kaajakari, T. Mattila, A. Oja, and H. Seppa, "Nonlinear limits for single-crystal silicon microresonators," J. Microelectromech. Syst., vol. 13, no. 5, pp. 715-724, Oct. 2004.

[36] S. Timoshenko, Vibration Problems in Engineering, 4th ed. Hoboken, NJ, USA: Wiley, 1974.

[37] V. Kaajakari, Practical MEMS: Analysis and Design of Microsystems, MEMS Sensors, 1st ed. Las Vegas, NV, USA: Small Gear Publishing, 2009. 


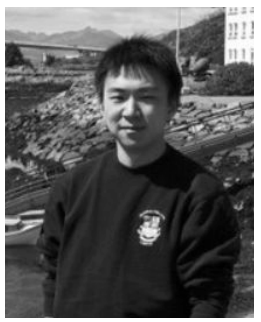

Chun Zhao (S'14) received the B.Eng. degree in measurement and control technology and instrument from the Huazhong University of Science and Technology, Wuhan, China, in 2009, and the M.Sc. degree in analog and digital IC design from Imperial College London, London, U.K. in 2011.

$\mathrm{He}$ is currently pursuing the Ph.D. degree in microelectronics with the Nano Research Group, School of Electronics and Computer Science, University of Southampton, Southampton, U.K. His current research interests include microelectromechanical systems, miniature sensing devices, microresonators, and interface circuit for sensors design.

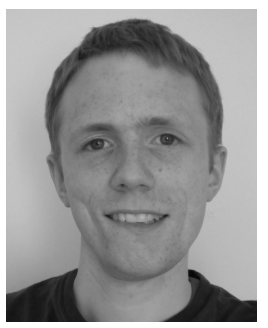

Graham S. Wood (M'10) received the M.Eng. degree in electronics and electrical engineering, and the M.Sc. degree by research in microelectronics from the University of Edinburgh, Edinburgh, U.K., in 2008 and 2011, respectively. $\mathrm{He}$ is currently pursuing the Ph.D. degree in microelectronics with the Nano Research Group, School of Electronics and Computer Science, University of Southampton, Southampton, U.K.

He was a Research Associate with the Scottish Microelectronics Center, Institute for Integrated Micro and Nano Systems, School of Engineering, University of Edinburgh, from 2008 to 2010, where he conducted research concerning the actuation and sensing of $\mathrm{SiC}$ microelectromechanical systems (MEMS) resonators for high-frequency RF applications. His current research interests include the use of mode localization in electrically coupled MEMS resonators for sensor applications.

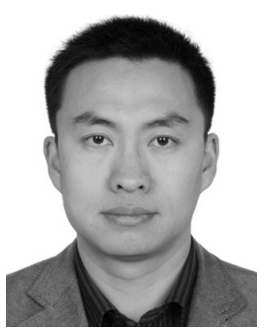

Jianbing Xie (M'13) received the B.S., M.S., and Ph.D. degrees from Northwestern Polytechnical University (NPU), Xi'an, China, in 2003, 2006, and 2010, respectively, all in mechanical engineering.

$\mathrm{He}$ is currently an Associate Professor with the Micro and Nano Electromechanical Systems Laboratory, NPU. His research interests include MEMS inertial sensors and micromachining process.

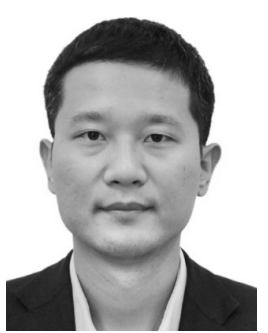

Honglong Chang (M'04) received the B.S., M.S., and Ph.D. degrees from Northwestern Polytechnical University (NPU), Xi'an, China, in 1999, 2002, and 2005, respectively, all in mechanical engineering.

He was a Visiting Associate (Faculty) with the Micromachining Laboratory, California Institute of Technology, Pasadena, CA, USA, from 2011 to 2012. He is currently a Professor with the Micro and Nano Electromechanical Systems Laboratory, NPU, where he is also the Chair of the Department of Microsystem Engineering.

He has authored over 26 international journal papers and over 20 international conference papers in the Micro and Electromechanical Systems (MEMS) field. His research interests include MEMS inertial sensors and MEMS design tools.

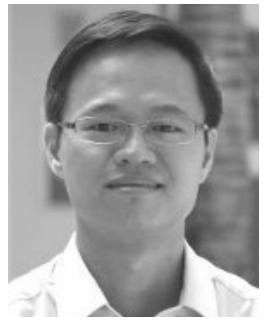

Suan Hui Pu (S'09-M'11) received the M.Eng. degree in mechanical engineering, and the $\mathrm{Ph} . \mathrm{D}$. degree in electrical and electronic engineering from Imperial College London, London, U.K., in 2006 and 2010, respectively.

$\mathrm{He}$ was a Product Engineer with Infineon Technologies (Kulim) Sdn. Bhd., from 2010 to 2012, working on product yield enhancement for a bipolar-CMOS-DMOS process technology. He is currently an Assistant Professor with the University of Southampton-Malaysia Campus, and a Visiting Academic with the Nano Research Group, Electronics and Computer Science, University of Southampton, U.K. He has authored over 16 peer-reviewed papers in international journals and conferences. His current research interests include MEMS/NEMS sensors and actuators, graphene, nanocrystalline graphite, and wearable technology.

Dr. Pu is currently serving as a Technical Committee Member of the IEEE Electronics Packaging Technology Conference and a Reviewer of the IEEE JOURNAL OF MICROELECTROMECHANICAL SYSTEMS.

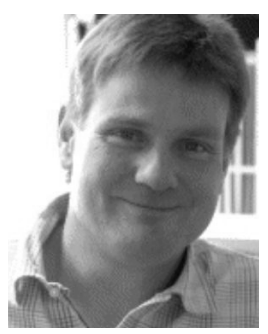

Michael Kraft received the Dipl.-Ing. degree in electrical and electronics engineering from Friedrich Alexander Universität Erlangen Nürnberg, Erlangen, Germany, in 1993, and the Ph.D. degree from Coventry University, Coventry, U.K., in 1997.

He was an academic with the School of Electronics and Computer Science, University of Southampton, Southampton, U.K, from 1999 to 2012, where he acted as the Director with the Southampton Nanofabrication Center, Southampton. He was with the Berkeley Sensors and Actuator Center, University of California, Berkeley, CA, USA, working on integrated microelectromechanical systems (MEMS) gyroscopes. He has focused on novel micro- and nanofabrication techniques, microsensors, and actuators and their interface circuits, and in particular, capacitive sensors. He was with the Fraunhofer Institute for Microelectronic Circuits and Systems, Duisburg, Germany, from 2012 to 2014, where he was the Head of the Department of Micro- and Nanosystems with a focus on fully integrated microsensors and biohybrid systems. He is currently a Professor of Micro- and Nanosystems with the University of Liege, Belgium. Concurrently, he held the Professorial Chair (W3) of Integrated Micro- and Nanosystems at the University of Duisburg-Essen. $\mathrm{He}$ has authored or co-authored over 160 peer-reviewed journal and conference papers. He has contributed to three textbooks on MEMS and an edited MEMS for Aerospace and Automotive Applications. His current research interests include MEMS and nanotechnology ranging from process development to system integration of MEMS and nanodevices.

Dr. Kraft serves on several steering and technical committees of international conferences, such as the IEEE Sensors, Eurosensors, and the Micromechanics and Microsystems Europe Workshop. 